\title{
FLAT SUBMODULES OF FREE MODULES OVER COMMUTATIVE BEZOUT RINGS
}

\begin{abstract}
K. SAMEI
A ring is called Bezout if every finitely generated ideal is principal. We show that every ideal of a commutative Bezout ring $R$ is tlat if and only if every submodule of a free $R$-module is flat. Using this theorem we obtain Neville's theorem.
\end{abstract}

\section{INTRODUCTION}

Neville has proved that the topological space $X$ is an $F$ space if and only if every ideal of $C(X)$ is flat, or if and only if every submodule of a free $C(X)$-module is flat. This theorem is the main result in [4]. In this paper we define quasi-torsion-free modules, and when $R$ is a commutative Bezout ring we show an $R$-module is quasi-torsion-free if and only if it is flat. We also show that every ideal of $R$ is flat if and only if every submodule of a free $R$-module is flat. In Section 3, we prove Neville's theorem using these theorems.

We need to review briefly some standard terminology. In this paper $R$ is always a commutative ring with identity and modules are unital. An $R$-module is flat if the tensor product is an exact functor. An ideal $I$ of a ring $R$ is called pure if for every $a \in I$, there exists $b \in I$, such that $a=a b$.

We denote by $\operatorname{Max}(R)$ the spectrum of maximal ideals of $R$. We say $R$ is semiprimitive if $\cap \operatorname{Max}(R)=(0)$. For any ideal $I$ of $R$ and $a \in R$, we set

$$
\mathrm{M}(a)=\{M \in \operatorname{Max}(R): a \in M\} \text { and } \mathrm{M}(I)=\{M \in \operatorname{Max}(R): I \subseteq M\} .
$$

Then the sets $\mathrm{M}(I)=\bigcap_{a \in I} \mathrm{M}(a)$, where $I$ is an ideal of $R$, satisfy the axioms for the closed sets of a topology on $\operatorname{Max}(R)$, called the Stone topology, see [3, 7M].

Throughout, $X$ will denote a completely regular and Hausdorff space and $C(X)$ denotes the ring of continuous real-valued functions on $X$. Two sets $E$ and $F$ are completely separated if there exists some $f \in C(X)$ such that $f=0$ on $E$ and $f=1$ on $F$. The cozero set of a function $f \in C(X)$ is the set $\operatorname{coz}(f)=\{x \in X: f(x) \neq 0\}$. A space $X$ is an $F$ space if disjoint cozero sets are always completely separated. Several equivalent conditions for $F$ spaces are given in [3, Theorem 14.25]; in particular $X$ is an $F$ space if and only if $C(X)$ is a Bezout ring. The reader is referred to [3] for undefined terms and notations.

\footnotetext{
Received 6th September, 2004
}

This research was in part supported by a grant from IPM (No 83130029).

Copyright Clearance Centre, Inc. Serial-fee code: 0004-9727/05 \$A2.00+0.00. 


\section{Submodules of Free Modules}

The following lemma is proved in [1].

LEMMA 2.1. In a ring $R$, every principal ideal is tlat if and only if for each $a \in R, \operatorname{Ann}(a)$ is a pure ideal.

REMARK. Let $R$ be a ring. Suppose

$$
0 \longrightarrow K \longrightarrow F \stackrel{\phi}{\longrightarrow} A \longrightarrow 0
$$

is an exact sequence of $R$-modules, where $F$ is flat. If $A$ is flat, then for every principal ideal $I=(r)$ of $R$ we have: $K \cap F I=K I$, see [5, Theorem 3.55]. It is easy to see that for any $x \in F, x r \in K \cap F I$ implies that $x r=k r$, for some $k \in K$.

By redefining the concept of torsion, we can say something interesting about flat modules.

Definition: Consider the exact sequence of $R$-modules

$$
0 \longrightarrow K \longrightarrow F \stackrel{\phi}{\longrightarrow} A \longrightarrow 0
$$

where $F$ is a flat submodule of a free module. A $R$-module $A$ is quasi-torsion-free relative to the exact sequence (1), if the following is true: whenever $r \in R, x \in A$ and $r x=0$, there are $x^{\prime} \in F$ and $k \in K$ such that $\phi\left(x^{\prime}\right)=x$ and $r x^{\prime}=r k$.

The independence of the notion of quasi-torsion-free from the exact sequence (1) follows from the following lemma.

LEMMA 2.2. Let $R$ be a ring. If an $R$-module is quasi-torsion-free relative to one exact sequence, it is quasi-torsion-free relative to every exact sequence.

Proof: Suppose that $A$ is quasi-torsion-free relative to the exact sequence

$$
0 \longrightarrow K_{2} \longrightarrow F_{2} \stackrel{\phi_{2}}{\longrightarrow} A \longrightarrow 0
$$

where $F_{2}$ is a flat submodule of a free module. Use the fact that every module is a quotient of a free module to find a free module $F_{1}$ and an onto map $\psi_{1}: F_{1} \longrightarrow F_{2}$. Define the following diagram of exact sequences

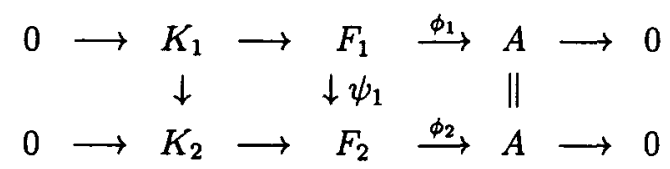

by letting $\phi_{1}=\phi_{2} \circ \psi_{1}$ and $K_{1}=\operatorname{Ker}\left(\phi_{1}\right)$. Consider the exact sequence

$$
0 \longrightarrow K_{3} \longrightarrow F_{1} \stackrel{\psi_{1}}{\rightarrow} F_{2} \longrightarrow 0
$$

Suppose $r x=0$, where $r \in R$ and $x \in A$. Then there exist $x_{2} \in F_{2}$ and $k_{2} \in K_{2}$ such that $\phi_{2}\left(x_{2}\right)=x$ and $r x_{2}=r k_{2}$. Let $x_{1} \in F_{1}$ and $k_{1} \in K_{1}$ be such that $\psi_{1}\left(x_{1}\right)=x_{2}$ and 
$\psi_{1}\left(k_{1}\right)=k_{2}$. Therefore $r\left(x_{1}-k_{1}\right) \in K_{3}$. Therefore by the above remark, $r\left(x_{1}-k_{1}\right)=r k_{3}$, some $k_{3} \in K_{3}$. Now $K_{3} \subseteq K_{1}$, so $k_{1}+k_{3} \in K_{1}$. Thus we have $\phi_{1}\left(x_{1}\right)=\phi_{2} \circ \psi_{1}\left(x_{1}\right)=x$ and $r x_{1}=r\left(k_{1}+k_{3}\right)$. Hence $A$ is quasi-torsion-free relative to the top exact sequence. We note that the middle term of the top exact sequence is free. Now suppose that

$$
0 \longrightarrow K \longrightarrow F \stackrel{\phi}{\longrightarrow} A \longrightarrow 0
$$

is an arbitrary exact sequence of $R$-modules, where $F$ is a flat submodule of a free module. By the projectivity of free modules, there exists the following commutative diagram:

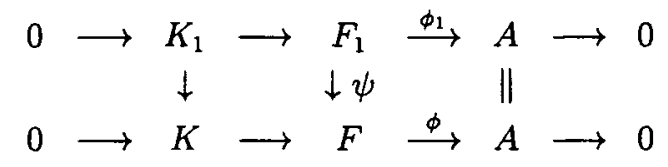

As in the above proof, it follows that $A$ is quasi-torsion-free relative to (2). This means that $A$ is quasi-torsion-free relative to every exact sequence.

THEOREM 2.3. Let $R$ be a ring. Then every flat $R$-module is quasi-torsion-free. If $R$ is Bezout, every quasi-torsion-free $R$-module is flat.

Proof: Suppose that $A$ is a $R$-module and consider the exact sequence

$$
0 \longrightarrow K \longrightarrow F \stackrel{\phi}{\longrightarrow} A \longrightarrow 0
$$

where $F$ is a submodule of a free module. First we claim that $A$ is quasi-torsion-free if and only if $K \cap F J=K J$, for all principal ideals $J$. Let $K \cap F J=K J$, for all principal ideals $J$ and let $r \in R, x \in A$ and $r x=0$, then there exists $x^{\prime} \in F$ such that $\phi\left(x^{\prime}\right)=x$. So $r x^{\prime} \in K \cap F J$, where $J=(r)$. Hence by the remark, there exists $k \in K$ such that $r x^{\prime}=r k$ and this implies that $\therefore$ is quasi-torsion-free. Conversely, let $A$ be quasi-torsion-free and $r x \in K \cap F J$, where $r \in R$ and $x \in F$. Then $r \phi(x)=0$, so there are $x^{\prime} \in F$ and $k \in K$ such that $\phi\left(x^{\prime}\right)=\phi(x)$ and $r x^{\prime}=r k$. Since $x=x^{\prime}+k^{\prime}$, for some $k^{\prime} \in K$, then $r x=r\left(k+k^{\prime}\right)$. Therefore $r x \in K J$ and this proves the claim. Now the proof follows from [5, Theorem 3.55].

We come now to the main result of this section. But we first need the following lemma.

LemMA 2.4. Let $R$ be a ring and let $A$ be a submodule of $\coprod_{j \in T} R$. Let $\pi_{j}$ be the canonical projection map onto the $j$ th coordinate, and assume the ideal $\pi_{n}(A)=I_{n}$ is principal with generator $a_{n}$. Then the exact sequence

$$
0 \longrightarrow K_{n} \stackrel{i}{\longrightarrow} A \stackrel{\pi_{n}}{\longrightarrow} I_{n} \longrightarrow 0
$$

splits if and only if there exists $x_{n} \in A$ with $\pi_{n}\left(x_{n}\right)=a_{n}$ and $\operatorname{Ann}\left(a_{n}\right) \subseteq \operatorname{Ann}\left(x_{n}\right)$.

Proof: Assume that the above exact sequence splits. Let $\psi_{n}: I_{n} \rightarrow A$ be the splitting homomorphism and let $x_{n}=\psi_{n}\left(a_{n}\right)$. If $a \in I_{n}$, then $\psi_{n}(a)=\psi_{n}\left(b a_{n}\right)$ 
$=b \psi_{n}\left(a_{n}\right)=b x_{n}$, for any $b$ such that $b a_{n}=a$. In other words, $b a_{n}=0$ implies that $b x_{n}=0$, for any $b \in R$. Consequently, $\operatorname{Ann}\left(a_{n}\right) \subseteq \operatorname{Ann}\left(x_{n}\right)$.

Conversely, suppose that there exists $x_{n} \in A$ such that $\pi_{n}\left(x_{n}\right)=a_{n}$ and $\operatorname{Ann}\left(a_{n}\right)$ $\subseteq \operatorname{Ann}\left(x_{n}\right)$. Define the splitting homomorphism $\psi_{n}: I_{n} \rightarrow A$ by $\psi_{n}\left(b a_{n}\right)=b x_{n}$. Now if $b a_{n}=c a_{n}$, then $b-c \in \operatorname{Ann}\left(a_{n}\right) \subseteq \operatorname{Ann}\left(x_{n}\right)$, so $\psi_{n}$ is well defined. Clearly, $\psi_{n}$ is a module homomorphism. Finally, $\pi_{n} \circ \psi_{n}\left(b a_{n}\right)=b \pi_{n} \circ \psi_{n}\left(a_{n}\right)=b \pi_{n}\left(x_{n}\right)=b a_{n}$, so $\psi_{n}$ is indeed a splitting homomorphism.

THEOREM 2.5. Let $R$ be a Bezout ring. Then every principal ideal of $R$ is flat if and only if every finitely generated submodule of a free $R$-module is flat.

Proof: Suppose that every principal ideal of $R$ is flat. Let $A$ be a finitely generated submodule of a free module. Then $A$ can be embedded in a finitely generated free module. So without loss of generality $A \subseteq \bigsqcup_{1}^{n} R$. The proof is by induction on $n$. If $n=1$ then $A$ is principal ideal, and so is flat by the hypothesis. Suppose $n>1$ and the theorem has been proof for all finitely generated modules contained in $\coprod_{1}^{n-1} R$. Let $F_{n}$ be the free module $\coprod_{1}^{n} R$. Let $\pi_{j}$ and $I_{n}$ be as in the lemma. Since $R$ is Bezout and $I_{n}$ is finitely generated, $I_{n}=\left(a_{n}\right)$, for some $a_{n} \in R$. Consider the homomorphism $\phi: F_{n} \rightarrow F_{n}$ defined by $\phi\left(b_{1}, \ldots, b_{n-1}, b_{n}\right)=\left(b_{1}, \ldots, b_{n-1}, b_{n} a_{n}\right)$. We want to consider a suitable submodule $B$ of $F_{n}$ (or rather $\phi^{-1}(A)$ ), so that we can apply Lemma 2.4 with $\pi_{n}\left(x_{n}\right)=1$. Since $A$ is finitely generated, there exists a finitely generated submodule $B$ of $F_{n}$ such that $\phi(B)=A$. Since $a_{n} \in \pi_{n}(A)$, we may assume without loss of generality that there exists $x_{n}^{\prime} \in B$ such that $\pi_{n}\left(x_{n}^{\prime}\right)=1$ (If no such $x_{n}^{\prime}$ exists, consider any $x^{\prime}=\left(b_{1}, \ldots, b_{n-1}, b_{n}\right) \in B$ such that $\pi_{n} \circ \phi\left(x^{\prime}\right)=a_{n}$, that is, $a_{n} b_{n}=a_{n}$. Let $x_{n}^{\prime}=\left(b_{1}, \ldots, b_{n-1}, 1\right)$ and enlarge $B$ to include $x_{n}^{\prime}$ ). Clearly, $\pi_{n}(B)=R$. Consider the exact sequence

$$
0 \longrightarrow L_{n} \longrightarrow B \stackrel{\pi_{n}}{\longrightarrow} R \longrightarrow 0 .
$$

Trivially, the hypothesis of Lemma 2.4 is satisfied, and so $B=L_{n} \oplus R$. Clearly $L_{n}=\left\{\left(b_{1}, \ldots, b_{n-1}, 0\right) \in B\right\}$ is embedable in $\coprod_{1}^{n-1} R$, so that $L_{n}$ is flat by the inductive hypothesis. Thus $B$ is flat. Now consider the exact sequence

$$
0 \longrightarrow K \longrightarrow B \stackrel{\phi}{\longrightarrow} A \longrightarrow 0
$$

We shall prove that $A$ is flat by proving that $A$ is quasi-torsion-free relative to the exact sequence (3). First note that the middle term $B$ is a flat submodule of the free module $F_{n}$. Now assume that $x \in A, r \in R$ and $r x=0$. We must find $x^{\prime} \in B$ and $k \in K$ such that $\phi\left(x^{\prime}\right)=x$ and $r x^{\prime}=r k$. Let $x^{\prime}=\left(b_{1}, \ldots, b_{n-1}, b_{n}\right) \in B$ be such that $\phi\left(x^{\prime}\right)=\left(b_{1}, \ldots, b_{n-1}, b_{n} a_{n}\right)=x$. Therefore $\left(r b_{1}, \ldots, r b_{n-1}, r b_{n} a_{n}\right)=0$, that is,

$$
r \in \operatorname{Ann}\left(b_{1}\right) \cap \cdots \cap \operatorname{Ann}\left(b_{n-1}\right) \cap \operatorname{Ann}\left(b_{n} a_{n}\right)
$$


Since $R$ is Bezout, then

$$
\operatorname{Ann}\left(b_{1}\right) \cap \cdots \cap \operatorname{Ann}\left(b_{n-1}\right) \cap \operatorname{Ann}\left(b_{n} a_{n}\right)=\operatorname{Ann}(b),
$$

for some $b \in R$. According to Lemma 2.1, $\operatorname{Ann}(b)$ is pure, hence there exists $c \in \operatorname{Ann}(b)$ such that $r=r c$. Set $k=c x^{\prime}$. Clearly $k \in B, r x^{\prime}=r k$ and $\phi(k)=\phi\left(c b_{1}, \ldots, c b_{n-1}, c b_{n} a_{n}\right)=0$, that is, $k \in K$. Thus $A$ is quasi-torsion-free relative to the exact sequence (3). Hence $A$ is flat, by Theorem 2.3 .

It is well-known that a $R$-module $A$ is flat if and only if every finitely generated submodule of $A$ is flat. Thus we have:

COROLlary 2.6. Let $R$ be a Bezout ring. Then every ideal of $R$ is flat if and only if every submodule of a free $R$-module is flat.

\section{Gelfand RINGS}

The purpose of this section is to prove Neville's theorem, by the theorems of the previous section. We first give some results about semiprimitive Gelfand rings.

A ring $R$ is called Gelfand ( $p m$-ring) if every prime ideal of $R$ is contained in a unique maximal ideal. When the Jacobson radical and the nilradical of ring $R$ coincide, DeMarco and Orsatti [2] show that $R$ is Gelfand if and only if $\operatorname{Max}(R)$ is Hausdorff; and if and only if $\operatorname{Spec}(R)$ is normal (in general, not Hausdorff). This class of rings contains the classes of regular ring, local rings, zero-dimension rings and $C(X)$.

DEFinition. Two subsets $E$ and $F$ of $\operatorname{Max}(R)$ are said to be almost separated in $\operatorname{Max}(R)$ if there exists $a \in R$ such that $E \subseteq \mathrm{M}(a)$ and $F \subseteq \mathrm{M}(a-1)$.

PROPOSITION 3.1. Let $R$ be a ${ }^{\wedge m i p r i m i t i v e ~ r i n g, ~ t h e n ~ e v e r y ~ p r i n c i p a l ~ i d e a l ~}$ in $R$ is flat if and only if for any non-zero $a, b \in R, \operatorname{Max}(R)-\mathrm{M}(a)$ and $\operatorname{Max}(R)-\mathrm{M}(b)$ are almost separated whenever $a b=0$.

Proof. Suppose every principal ideal is flat and $a, b \in R$ such that $a b=0$. By Lemma 2.1, $\operatorname{Ann}(a)$ is pure, so there exists $c \in \operatorname{Ann}(a)$ such that $b c=b$. Hence $a c=0, b(c-1)=0$. Thus

$$
\operatorname{Max}(R)-\mathrm{M}(a) \subseteq \mathrm{M}(c), \operatorname{Max}(R)-\mathrm{M}(b) \subseteq \mathrm{M}(c-1) .
$$

Conversely, let $a \in R$. We want to show that $\operatorname{Ann}(a)$ is pure. Let $b \in \operatorname{Ann}(a)$. If $b=0$, then there exists $b=0 \in \operatorname{Ann}(a)$ such that $b^{2}=b=0$. So we can assume $b \neq 0$. Because $a b=0, \operatorname{Max}(R)-\mathrm{M}(a)$ and $\operatorname{Max}(R)-\mathrm{M}(b)$ are almost separated. Hence there exists $c \in R$ such that

$$
\operatorname{Max}(R)-\mathrm{M}(a) \subseteq \mathrm{M}(c) \text { and } \operatorname{Max}(R)-\mathrm{M}(b) \subseteq \mathrm{M}(c-1)
$$

Thus $a c=0$ and $b c=b$. Hence $\operatorname{Ann}(a)$ is pure. 
LEMmA 3.2. Let $R$ be a Gelfand ring, then the subsets $E$ and $F$ of $\operatorname{Max}(R)$ are completely separated if and only if they are almost separated in $\operatorname{Max}(R)$.

Proof: Assume $E$ and $F$ are completely separated in $\operatorname{Max}(R)$. So $\operatorname{cl} E \cap \operatorname{cl} F=\emptyset$. Hence there exists the ideals $I$ and $J$ such that $\operatorname{cl} E=\mathrm{M}(I)$ and $\operatorname{cl} F=\mathrm{M}(J)$. We claim that $I+J=R$. Otherwise there exists $M \in \operatorname{Max}(R)$ such that $I+J \subseteq M$. So $M \in \mathrm{M}(I) \cap \mathrm{M}(J)$, and this is a contradiction. Therefore $a+b=1$, for some $a \in I$ and $b \in J$. Thus

$$
\mathrm{M}(I) \subseteq \mathrm{M}(a) \text { and } \mathrm{M}(J) \subseteq \mathrm{M}(a-1) .
$$

Conversely, Assume $E$ and $F$ are almost separated in $\operatorname{Max}(R)$. Then there exists $a \in R$ such that

$$
E \subseteq \mathrm{M}(a) \text { and } F \subseteq \mathrm{M}(a-1) \text {. }
$$

Thus by Urysohn's Lemma there exists the function $f: \operatorname{Max}(R) \longrightarrow R$ such that

$$
f(\mathrm{M}(a))=0 \text { and } f(\mathrm{M}(a-1))=1 .
$$

This shows that $E$ and $F$ are completely separated.

The following result is a generalisation of [1, Theorem 4].

THEOREM 3.3. Let $R$ be a semiprimitive Gelfand ring. Then every principal ideal in $R$ is flat if and only if for any non-zero $a, b \in R, \operatorname{Max}(R)-M(a)$ and $\operatorname{Max}(R)$ $-M(b)$ are completely separated whenever $a b=0$.

Proof: It is obvious from Proposition 3.1 and Lemma 3.2.

LEMMA 3.4. $X$ is an $F$ space if and only if for any non-zero $f, g \in C(X)$, $\operatorname{Max}(C(X))-\mathrm{M}(f)$ and $\operatorname{Max}(C(X))-\mathrm{M}(g)$ are completely separated whenever $f g=0$.

ProOF: We consider the map $\psi: \beta X \rightarrow \operatorname{Max}(C(X))$ such that $\forall x \in \beta X, \psi(x)$ $=M^{x}$, where $\beta X$ is the stone-Čech compactification of $X$. It is well-known that $\psi$ is a homeomorphism, and hence $\operatorname{Max}(C(X)) \cong \beta X$, see [3, Section 6]. Therefore by [3, Theorem 7.3], for any $f \in C(X)$, we have:

$$
\psi(\underset{\beta X}{\mathrm{cl}} \mathrm{Z}(f))=\left\{M^{x} \in \operatorname{Max}(C(X)): f \in M^{x}\right\}=\mathrm{M}(f) .
$$

Consequently, $X$ is an $F$ space if and only if for any non-zero $f, g \in C(X), X-\mathrm{Z}(f)$ and $X-\mathrm{Z}(g)$ are completely separated whenever $f g=0$ (see $[3,14 \mathrm{~N} .4]$ ); if and only if for any non-zero

$$
f, g \in C(X), \underset{\beta X}{\operatorname{cl}}(X-\mathrm{Z}(f))=\beta X-\underset{\beta X}{\operatorname{cl} \mathrm{Z}}(f)
$$

and

$$
\underset{\beta X}{\mathrm{cl}}(X-\mathrm{Z}(g))=\beta X-\underset{\beta X}{\operatorname{cl}} \mathrm{Z}(g)
$$

are completely separated in $\beta X$, whenever $f g=0$; and if and only if for any non-zero

$$
f, g \in C(X), \operatorname{Max}(C(X))-\mathrm{M}(f)
$$


and

$$
\operatorname{Max}(C(X))-\mathrm{M}(g)
$$

are completely separated whenever $f g=0$.

THEOREM 3.5. The following are equivalent:

(1) $X$ is an $F$ space.

(2) every submodule of a free $C(X)$-module is flat.

(3) every ideal of $C(X)$ is flat.

Proof: To prove (1) $\Longrightarrow(2)$ suppose $X$ is an $F$ space, then $C(X)$ is a Bezout ring, by [3, Theorem 14.25]. Thus (2) follows from Corollary 2.6, Theorem 3.3 and Lemma 3.4. It is trivial to show (1) $\Longrightarrow(2)$. Finally (3) $\Longrightarrow(1)$ follows from Theorem 3.3 and Lemma 3.4 .

\section{REFERENCES}

[1] H. Al-Ezeh, M.A. Natsheh and Hussein, 'Some properties of the ring of continuous functions', Arch. Math. (Basel) 51 (1988), 60-64.

[2] G. De Marco and A. Orsatti, 'Commutative rings in which every prime ideal is contained in a unique maximal ideal', Proc. Amer. Math. Soc. 30 (1971), 459-466.

[3] L. Gillman and M. Jerison, Rings of continuous functions (Springer-Verlag, Berlin, Heidelberg, New York, 1976).

[4] C.W. Neville, 'Flat $C(X)$-modules and $F$ spaces', Math. Proc. Cambridge Philos. Soc. 106 (1989), 237-244.

[5] J. Rotman, An introduction to homological algebra, Pure and Applied Maths 148 (Academic. Press, New York, London, 1979).

Department of Mathematics

Bu Ali Sina University

Hamedan

Iran

e-mail: ipm@samei.ir
Institute for studies in Theoretical Physics and Mathematics (IPM)

Tehran

Iran. 